\title{
Identification of a metabolomic signature associated with feed efficiency in beef cattle
}

\author{
Francisco José Novais ${ }^{1}$, Pedro Ratto Lisboa Pires ${ }^{1}$, Pâmela Almeida Alexandre ${ }^{1}$, Robert A Dromms², \\ Amadeu Hoshi Iglesiass ${ }^{3}$, José Bento Sterman Ferraz ${ }^{1}$, Mark Philip-Walter Styczynski ${ }^{2}$ and Heidge Fukumasu ${ }^{1 *}$ (10
}

\begin{abstract}
Background: Ruminants play a great role in sustainable livestock since they transform pastures, silage, and crop residues into high-quality human food (i.e. milk and beef). Animals with better ability to convert food into animal protein, measured as a trait called feed efficiency (FE), also produce less manure and greenhouse gas per kilogram of produced meat. Thus, the identification of high feed efficiency cattle is important for sustainable nutritional management. Our aim was to evaluate the potential of serum metabolites to identify FE of beef cattle before they enter the feedlot.
\end{abstract}

Results: A total of 3598 and $4210 \mathrm{~m} / \mathrm{z}$ features was detected in negative and positive ionization modes via liquid chromatography-mass spectrometry. A single feature was different between high and low FE groups. Network analysis (WGCNA) yielded the detection of 19 and 20 network modules of highly correlated features in negative and positive mode respectively, and 1 module of each acquisition mode was associated with RFI ( $r=0.55, P<0.05)$. Pathway enrichment analysis (Mummichog) yielded the Retinol metabolism pathway associated with feed efficiency in beef cattle in our conditions.

Conclusion: Altogether, these findings demonstrate the existence of a serum-based metabolomic signature associated with feed efficiency in beef cattle before they enter the feedlot. We are now working to validate the use of metabolites for identification of feed efficient animals for sustainable nutritional management.

Keywords: Residual feed intake, Nellore, Retinol, WGCNA

\section{Background}

The Food and Agriculture Organization of the United Nations estimates the world population will reach 9 billion people by 2050 and as a consequence, livestock production must double to meet the demand for food [1]. Sustainable livestock production is a field of intense research where ruminants play a great role since they can transform graze pastures, silage and high-fiber crop residues into high-quality human food (i.e. milk and meat) [2]. The goal is "sustainable intensification" [3], meaning increased productivity while reducing the environmental impacts. In this context, feed efficiency (FE) has a

\footnotetext{
* Correspondence: fukumasu@usp.br; http://orcid.org/0000-0002-3265-5090 ${ }^{1}$ Department of Veterinary Medicine, School of Animal Science and Food Engineering, University of Sao Paulo, Av. Duque de Caxias Norte n²25, Pirassununga 13635-900, Sao Paulo, Brazil

Full list of author information is available at the end of the article
}

particular importance, since it is directly related to productivity, greenhouse gas emission intensities, and resource use $[4,5]$.

Due to its importance, more than two dozen feed efficiency measurements have been proposed to select efficient animals and from those, residual feed intake (RFI) is considered one of the most effective methods $[6,7]$. As a complex trait, at least five major physiological mechanisms contribute to RFI variation: feed intake behavior, digestion, physical activity, thermoregulation and cell anabolism/catabolism [8]. Recently, our group proposed a new biological process associated with $\mathrm{FE}$ in beef cattle: increased hepatic inflammation in less efficient animals probably caused by altered lipid metabolism and/or increased bacterial infection associated with higher feed intake [9]. 
Metabolomics is the systems-scale study of low-molecular-weight biochemicals $(<1500 \mathrm{Da})$ involved in metabolism, including carbohydrates, lipids, amino acids, biogenic amines, and organic acids $[10,11]$. Due to the important role of metabolism across all biological processes, metabolomics studies have been increasingly used to understand physiological processes associated with economically important traits in livestock such as meat quality in pigs [12], milk production in dairy cattle [13-15] and growth in beef cattle [16]. Also, metabolomics has been applied to RFI studies, reporting blood metabolites in beef cattle during feedlot $[17,18]$.

Currently, there is an urgent need to develop new ways to predict FE in livestock, since the use of the commercially available genomic markers for genetic selection is not sensitive enough due to low to moderate heritability (ranging from 0.08 to 0.49 ) of the FE trait [19-22]. Therefore, we hypothesized there are specific serum metabolome signatures that predict feed efficiency in beef cattle before the feedlot which could be used for feed management of beef cattle. To this end, we used serum samples from a previous feeding trial with young Nellore bulls and performed a metabolomic approach on high and low feed efficient animals. The resulting data were used to investigate whether circulating metabolite levels could predict feed efficiency.

\section{Methods}

\section{Phenotypic data collection}

All animal procedures were approved by the Institutional Animal Care and Use Committee of the Faculty of Food Engineering and Animal Science at the University of Sao Paulo (protocol 14.1.636.74.1). The serum of 98 Nellore young bulls (16 to 20 months old and $376 \pm 29$ $\mathrm{kg} \mathrm{BW}$ ) born and raised in the University of Sao Paulo were collected 21 days prior to a 70-d feedlot. Briefly, the feeding-trial period was preceded by 21 days of adaptation to diet and location and before that, the animals were maintained in a single group on Brachiaria spp. pastures. On adaptation period, animals received corn silage (ad libitum), gradually replaced by trial diet (total mixed ration, including dry corn grain, corn silage, soybean, citrus pulp pellets, urea, calcareous, mineral salt and potassium chloride) offered at 8:00 h and 16:00 h. After the experiment, all animals were slaughtered following the guidelines of the Institutional Animal Care and Use Committee. More details regarding animals, diet and experimental design can be found in Alexandre et al. [9] and Mota et al. [23].

RFI was calculated as the difference between the expected and observed feed intake, considering the average metabolic weight (MBW) and ADG to predict DMI [6]. The 98 animals were ranked by RFI, and two groups of 8 animals each were selected for further analysis (total of
16 animals): high feed efficiency (HFE, low RFI) and low feed efficiency (LFE, high RFI). Sire and age effect on RFI were estimated by completely randomized design on linear model:

$$
Y i j k=\mu+\beta i+\beta k+e i j
$$

where $Y i j$ is the observation of $j$ th individual, son of ith sire, with $k$ age; $\mu$ is the general mean of the RFI; $\beta i$ is the sire effect; $\beta j$ is the age effect and eij is the random residual error, $\sim \operatorname{NID}\left(0, \sigma_{e}^{2}\right)$; and $\sigma_{e}^{2}$ is the residual variance. The phenotypic measures included: initial body weight (BWi), final body weight (BWF), dry matter intake (DMI), average daily gain (ADG), feed conversion ratio (FCR), residual feed intake (RFI), residual body weight gain (RWG), residual intake and body weight gain (RIG), initial ribeye area (REAi), final ribeye area (REAf) and gain of ribeye area (REAg). Normality of data was tested by the Shapiro-Wilk test. Student's t-test was applied to compare the groups for normally distributed variables and Mann-Whitney-Wilcoxon test for nonparametric variables using R STATS package. Results were considered significant when $p$-value $(P) \leq 0.05$. The RFI values were adjusted using regression model, in which the age was fitted as a covariate for network analysis (Additional file 1).

\section{Sample collection}

Serum samples were collected 21 days before the start of the feeding trial (before the adaptation period) by jugular venipuncture using vacutainer tubes. After $30 \mathrm{~min}$ at room temperature for clot formation, all samples were centrifuged at $3500 \times \mathrm{g}$ for $15 \mathrm{~min}$ at $4{ }^{\circ} \mathrm{C}$ and stored at $80{ }^{\circ} \mathrm{C}$ until further analysis, following the recommendations of Tuck et al. [24].

\section{LC-MS analysis}

Protein precipitation of serum samples was performed at $4{ }^{\circ} \mathrm{C}$ by adding methanol (1:4 serum: methanol) and vortexing for $120 \mathrm{~s}$ at $5000 \mathrm{rpm}$ [10]. The samples were then centrifuged at $16000 \mathrm{~g}$ for $4 \mathrm{~min}$ at room temperature, and the supernatants were dried in a vacuum centrifugal evaporator for $3 \mathrm{~h}$ at $30{ }^{\circ} \mathrm{C}$ and stored at $-20^{\circ} \mathrm{C}$ prior to analysis. The samples were reconstituted in $200 \mu \mathrm{L} \mathrm{H} \mathrm{H}_{2} \mathrm{O}$ and centrifuged at $12000 \mathrm{rpm}$ for $15 \mathrm{~min}$. The supernatants were transferred to analytical vials for analysis using a Xevo G2 XS quadrupole-time-of-flight mass spectrometer (Q-TOF-MS) in positive and negative modes (Waters Corporation, Milford, MA, USA). Chromatographic separation was performed by an Acquity I-Class UPLC system (Waters Corporation, Milford, MA, USA) using a Waters Acquity BEH C18 column $(2.1 \mathrm{~mm} \times 100 \mathrm{~mm}, 1.7 \mu \mathrm{m})$ (Waters Corporation, Milford, MA, USA) at $50{ }^{\circ} \mathrm{C}$. The injected sample volume 
was $5 \mu \mathrm{L}$. The mobile phase consisted of $0.1 \%$ formic acid-water (eluent A) and $0.1 \%$ formic acid-methanol (eluent $\mathrm{B}$ ). The gradient elution in positive mode was performed at a flow rate of $0.4 \mathrm{ml} / \mathrm{min}$, as follows: between 0 and 1 min $0 \%$ eluent $B ; 1-16$ min increasing up to $100 \%$ eluent $\mathrm{B} ; 16-20 \mathrm{~min}$ at $100 \%$ eluent $\mathrm{B}$ and 20 22 min decreasing back to $0 \%$ eluent $\mathrm{B}$. The elution flow rate was $0.36 \mathrm{ml} / \mathrm{min}$ in negative mode, with an elution gradient as follows: $0-2 \mathrm{~min} 0 \%$ eluent $\mathrm{B} ; 2-17 \mathrm{~min}$ increasing up to $100 \%$ eluent $\mathrm{B} ; 17-22 \mathrm{~min}$ at $100 \%$ eluent $\mathrm{B}$ and $22-24$ min decreasing back to $0 \%$ eluent $\mathrm{B}$.

The UPLC was connected to the electrospray ionization (ESI) interface, operating in negative and positive modes, with a capillary voltage of $-2.5 /+3 \mathrm{KV}$, source temperature of $150^{\circ} \mathrm{C}$, cone gas flow of $50 \mathrm{~L} / \mathrm{h}$, cone voltage of $40 \mathrm{~V}$, desolvation temperature of $550{ }^{\circ} \mathrm{C}$ and desolvation gas flow of $800 \mathrm{~L} / \mathrm{h}$. The spectra were collected at high resolution (mass resolving power $30,000 \mathrm{M} / \Delta \mathrm{M}$ at fwhm) from $100 \mathrm{~m} / \mathrm{z}$ (mass/charge ratio) to $1200 \mathrm{~m} / \mathrm{z}$, collected over $250 \mathrm{~ms}$ per spectrum in centroid mode. To avoid problems due to instrument drift, the sequence of samples was randomized and pooled quality-control samples $(\mathrm{QC})$ were injected periodically for use in downstream data processing and correction [10]. QC samples were prepared by pooling equal volumes of all samples; these samples were run after every four sample injections to provide a measurement of the stability and performance of the system.

\section{Data treatment and pre-processing}

LC-MS raw data were created and processed and using Waters MassLynx ${ }^{\mathrm{Tm}}$ (Waters Corporation, Milford, MA, USA) Software v4.1 and Progenesis QI (Nonlinear Dynamics, Newcastle, UK). Following the manufacturer's instructions, a reference run was automatically selected, and the precursor ion traces were processed for alignment, peak picking and normalization with default parameters. Locally estimated scatterplot smoothing (LOESS) signal correction based on QC samples was performed using MATLAB 2016 software with a script built for this purpose [25].

Afterward, a quality assurance (QA) step was used for analytical validation: variables with unacceptable reproducibility in QC samples (RSD $>20 \%$ in QCs or detected in less than $50 \%$ of QCs) and samples (detected in less than $90 \%$ of QC) were removed from the dataset [10]. The confidence scores of annotated metabolites are 2, meaning they have matches to a search database [26].

\section{Metabolomics data analysis}

Univariate and multivariate analyses were carried out using Metaboanalyst 4.0 Web Server [27]. Glog transformation [28] and auto-scaling [29] were applied. Differences between the groups were investigated using univariate (UA) and multivariate analysis (MA). For MA, principal component analysis (PCA) and partial least-square discriminant analysis (PLS-DA) were used for detection of outliers and to identify features potentially responsible for variation between the groups [29]. PLS-DA model quality was assessed using the goodness of fit $\left(\mathrm{R}^{2}\right)$ and goodness of prediction $\left(\mathrm{Q}^{2}\right)$ in cross-validation and using a permutation test with 2000 permutations [29]. For UA, t-test was used to identify differentially expressed features, then the $p$-values were corrected for multiple tests by Significance Analysis of Microarrays (SAM-FDR) method [30]. Features with SAM-FDR $q$-value $<0.05$ were considered different between groups.

\section{Network analysis}

Network and clustering analysis were performed using the Weighted Gene Co-expression Network Analysis (WGCNA) R package [31, 32]. Normalized data from positive and negative acquisition modes were used separately as described by Fukushima et al. [33], with a soft threshold of 3 , chosen using a scale-free topology criterion $\left(R^{2}=0.9\right)$. Modules containing at least 20 features were retained.

To select modules associated with FE, Pearson correlations between each module's "eigengene" and the RFI were calculated. The "eigengene" is the first principal component of a given module and a representative measure of its metabolic profile. (The term "gene" is used even for other data types, due to the development of WGCNA originally for the analysis of transcriptional data.) Modules with a module-trait relationship magnitude (correlation) $>0.5$ for RFI $(P \leq 0.05)$ were considered significant. Individual features were considered for further analysis only if they had module membership $(\mathrm{MM})>0.6(P<0.01)$ and gene significance $(\mathrm{GS})>0.5(P$ $<0.05)$. GS is defined as the association of features with RFI, and MM is defined as the correlation of the features with the module eigengene. High GS and MM scores indicate a feature is a central element of a module and is significantly associated with the trait [34].

\section{Metabolic pathway analysis}

Metabolic pathway analysis was performed using Mummichog software 1.0.9 with Bos taurus species (KEGG database) as reference [35]. Using default parameters for analyte prediction (mass accuracy $10 \mathrm{ppm}$ ) and for pathway enrichment analysis (1000 permutations). Features from UA with $P<0.01$ were used as input to mummichog to test for pathway enrichment compared to random data resampled from the reference list, yielding an empirical $p$-value per pathway. Pathways with corrected $q$-value $<0.05$ were considered significant. 


\section{Results}

We performed a 70-day feeding trial on 98 Nellore young bulls to evaluate their feed efficiency [9]. Based on the linear model (see Methods), there was no significant sire effect on RFI and the high feed efficient (HFE) and the low feed efficient (LFE) groups were statistically different $(P \leq 0.05)$ for all $\mathrm{FE}$ traits (feed conversion ratio (FCR), RFI, residual weight gain (RWG) and residual intake and weight gain (RIG), dry matter intake (DMI)) and also for average daily gain (ADG). There was also a significant difference for backfat thickness at the end of the experiment (BFTf, $P \leq 0.05$ ), which were greater in the LFE group (Table 1). Therefore, HFE animals in this experiment are more sustainable since they eat less, are leaner and have a better ADG than LFE animals.

\section{Metabolome profile and differential analysis}

After quality assurance processing, a total of 3598 and $4210 \mathrm{~m} / \mathrm{z}$ in negative and positive ionization modes, respectively, were used for parallel analyses. For Principal component analysis (PCA), no separation was observed for high and low FE animals in the first five principal components (Fig. 1), which explained 64.5 and $57 \%$ of total variance for negative and positive modes, respectively. PLS-DA was able to distinguish the two groups, but permutation and cross-validation analyses indicated the model was overfitted and thus not predictive (Fig. 1). The univariate analysis yielded one feature with different abundance between groups in positive mode. The spectra of mass-charge $183.1670 \mathrm{~m} / \mathrm{z}$ and retention time

Table 1 Descriptive statistics of high feed efficiency (HFE) and low feed efficiency (LFE) for phenotypic traits

\begin{tabular}{llll}
\hline Trait & HFE $( \pm$ SEM $)$ & LFE $( \pm$ SEM $)$ & $P$ value \\
\hline BWi $(\mathrm{kg})$ - & $410 \pm 16.03$ & $404.3 \pm 7.97$ & 0.64 \\
BWf $(\mathrm{kg}) \circ$ & $563.5 \pm 17.35$ & $525.8 \pm 9.87$ & 0.07 \\
DMI $(\mathrm{kg} / \mathrm{d})$ - & $10.38 \pm 0.39$ & $12.35 \pm 0.33$ & $<0.0001^{*}$ \\
ADG $(\mathrm{kg} / \mathrm{d})$ - & $2.194 \pm 0.15$ & $1.734 \pm 0.08$ & $0.0497^{*}$ \\
FCR - & $4.763 \pm 0.17$ & $7.3 \pm 0.29$ & $<0.0001^{*}$ \\
RFI $(\mathrm{kg} / \mathrm{d}) \circ$ & $-1.384 \pm 0.12$ & $1.791 \pm 0.12$ & $<0.0001^{*}$ \\
RWG $(\mathrm{kg} / \mathrm{d})$ - & $0.4325 \pm 0.07$ & $-0.3988 \pm 0.06$ & $<0.0001^{*}$ \\
RIG 0 & $1.815 \pm 0.10$ & $-2.188 \pm 0.13$ & $<0.0001^{*}$ \\
REAi $(\mathrm{cm} 2)$ - & $68.26 \pm 2.22$ & $67.23 \pm 1.95$ & 0.63 \\
REAf $(\mathrm{cm} 2)$ - & $84.94 \pm 2.58$ & $82.91 \pm 1.65$ & 0.64 \\
REAg $(\mathrm{cm} 2)$ - & $19.34 \pm 2.83$ & $15.69 \pm 1.77$ & 0.99 \\
BFTi $(\mathrm{mm}) \circ$ & $0.775 \pm 0.38$ & $1.975 \pm 0.46$ & 0.07 \\
BFTf $(\mathrm{mm})$ - & $2.975 \pm 0.67$ & $5.713 \pm 0.64$ & $0.0096^{*}$ \\
BFTg $(\mathrm{mm})$ - & $2.2 \pm 0.67$ & $3.738 \pm 0.37$ & 0.063 \\
\hline
\end{tabular}

$B W i$ initial body weight, $B W F$ final body weight, $D M I$ dry matter intake, $A D G$ average daily gain, $F C R$ feed conversion ratio, $R F I$ residual feed intake, $R W G$ residual body weight gain, $R I G$ residual intake and body weight gain, $R E A i$ initial ribeye area, $R E A f$ final ribeye area, $R E A g$ gain of ribeye area. ${ }^{*} P<0.05$. Student's t-test. OMann-Whitney-Wilcoxon Test v4.00 min on chromatography column (Fig. 2) has a $P<0.001$ (SAM-FDR $=0.03$ ) which is greater on HFE group. No significantly different $\mathrm{m} / \mathrm{z}$ were identified in negative mode.

\section{Pathway enrichment analysis}

Pathway enrichment analysis was performed to explore possible pathways involved in RFI phenotypic variation prior to the feedlot. Mummichog software identified the enrichment of retinol metabolic pathway $(P<0.05$; Table 2), as being associated with FE in positive mode with 2 pathway metabolites annotated in the data. The putative compounds hit included retinoate (C00777) and, either the isobaric compounds (molecular weight 284.4357): all-trans-Retinal (C00376) or 11-cis-Retinal (C02110) (Table 3).

\section{Weighted correlation network analysis}

We then used WGCNA co-expression analysis to identify clusters of analytes that may have a relationship with the feed efficiency. WGCNA identified 19 and 20 modules of highly correlated features in negative and positive mode, respectively.

One of these modules was significantly positively correlated with RFI (blue module from the negative mode, $r=0.55$, and $P=0.033$ ), indicating higher levels in LFE animals. The blue module contains 196 features (Fig. 3a), of which 65 were identified as important contributors to this module (Additional file 2). Using mummichog, three of these features were putatively annotated: (i) 6S,9R-Vomifoliol (compound KEGG C01760) (ii) 2,3, Dihydroflavone (compound C00766); (iii) Limonoate (compound C01593). The additional file 2 has information of the important features of blue module on negative mode, including mass-charge $(\mathrm{m} / \mathrm{z})$, retention time $(\mathrm{rt})$, feature significance to trait (GS), feature significance to module (FM), group with highest abundance, putative matched compound in KEGG and mass difference between feature and putative compound.

A second module was associated with RFI (blue module from the positive mode, $\mathrm{r}=0.55$, and $p$-value $=$ 0.033), also indicating higher levels in LFE animals. This module contains 112 features (Fig. 3b), of which 39 were identified as highly important contributors to this module (Additional file 3). Using mummichog, 5 compounds were annotated: (i) Phytanic acid (compound C01607); (ii) all-trans-Retinal (compound C00376); (iii) Progesterone (compound C00410); (iv) Limonoate (compound C01593); (v) Stearic acid (compound C01530). The additional file 3 has information of the important features of blue module on positive mode, including mass-charge $(\mathrm{m} / \mathrm{z})$, retention time $(\mathrm{rt})$, feature significance to trait (GS), feature significance to module (FM), group with highest abundance, putative matched compound in 
A
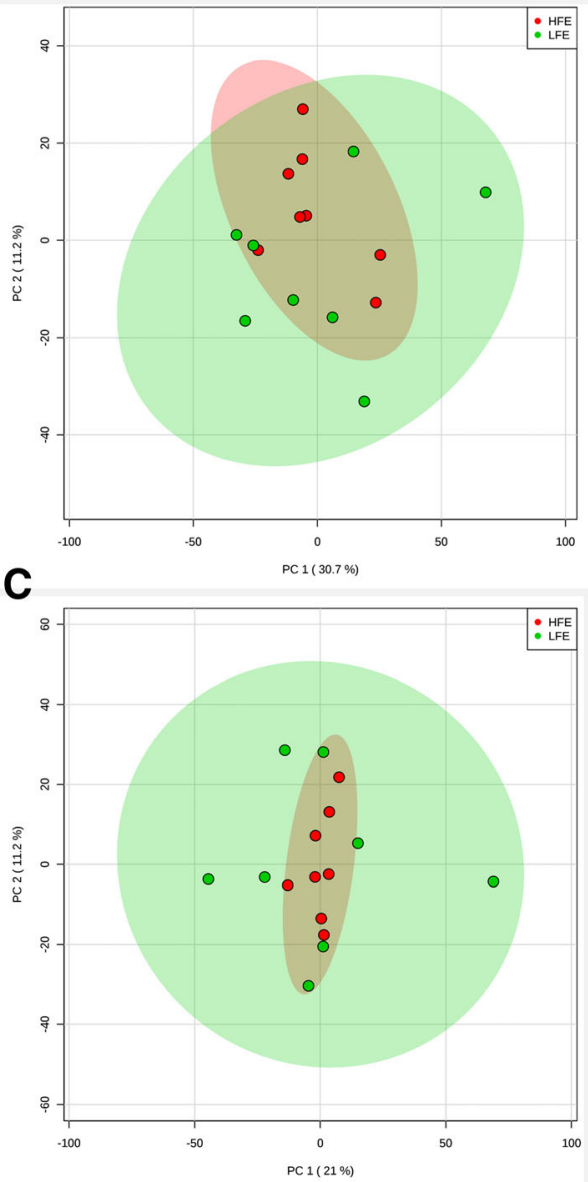

B
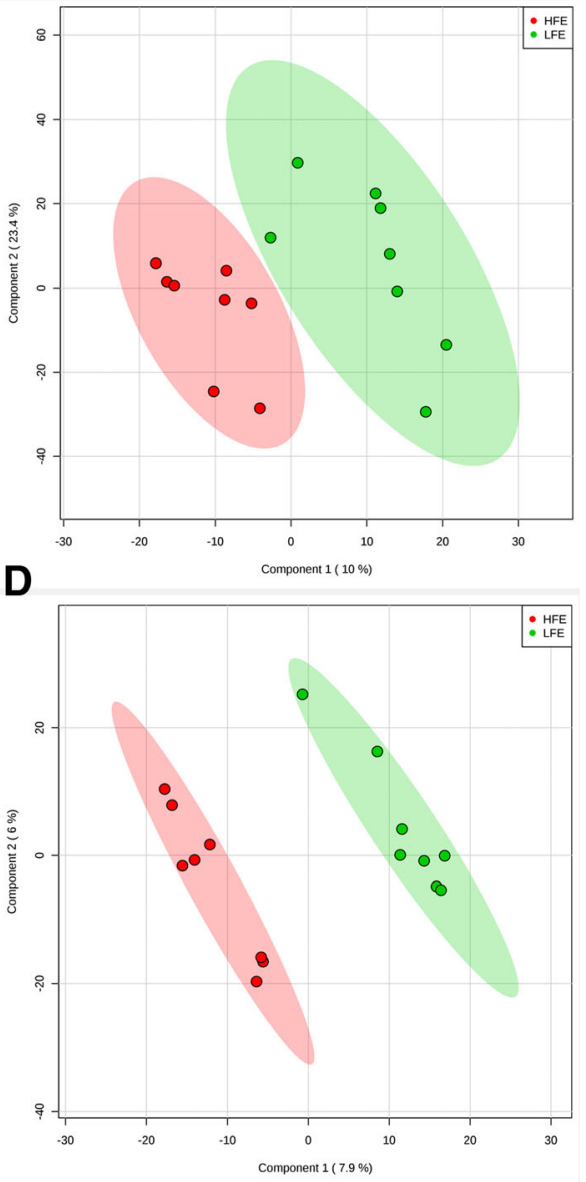

Fig. 1 PCA (a and $\mathbf{c}$, in negative and positive mode, respectively) and PLS-DA (b and $\mathbf{d}$, negative and positive mode, respectively) scores plots based on LC/MS data of serum samples from HFE (red) and LFE (green). The PLS-DA models discriminated between HFE and LFE groups (R² of 0.87 and 0.98 in negative and positive mode, respectively) but were not predictive $\left(\mathrm{Q}^{2}\right.$ of 0.08 and 0.15$)$. Considering a common heuristic for metabolomics data: $\mathrm{R}^{2}>0.8$ and $\mathrm{Q}^{2}>0.5$, the model was not overfitted. Consistent with this, a permutation test (2000 permutations) yielded $P$-values $>0.9$ in both modes

KEGG and mass difference between feature and putative compound.

\section{Discussion}

Brazilian cattle are mainly raised in pastures, but can also be kept in feedlot systems with diets composed of silage or other feedstuffs, such as high-fiber crop residues or grains (corn and soybean), to improve body weight gain before slaughter. We used serum samples collected before the feedlot period to search for a potential early metabolomic signature for FE, with the intent to support nutritional management decisions to improve productivity and sustainability of livestock. Thus, we performed an exploratory analysis using untargeted metabolomics coupled with bioinformatics and interpretation tools including Mummichog and WGCNA. We found one differentially expressed feature between
HFE and LFE animals in these conditions, but most importantly, we also found one enriched pathway and two sets of highly correlated features significantly associated with FE, which could be considered a potential molecular signature of FE in Nellore cattle before they enter the feedlot period.

A co-expression module associated with a phenotype provides significant promise for the development of a molecular signature, clearly more than a single statistically different feature between two conditions [36]. In our previous work, the co-expression gene modules and their gene ontology were far more important results than the differentially expressed genes [9]. In this context, the hepatic inflammatory response was associated with feed efficiency in cattle. Here, the WGCNA analysis indicated two modules of co-expressed features positively associated with RFI, with equal correlation, 


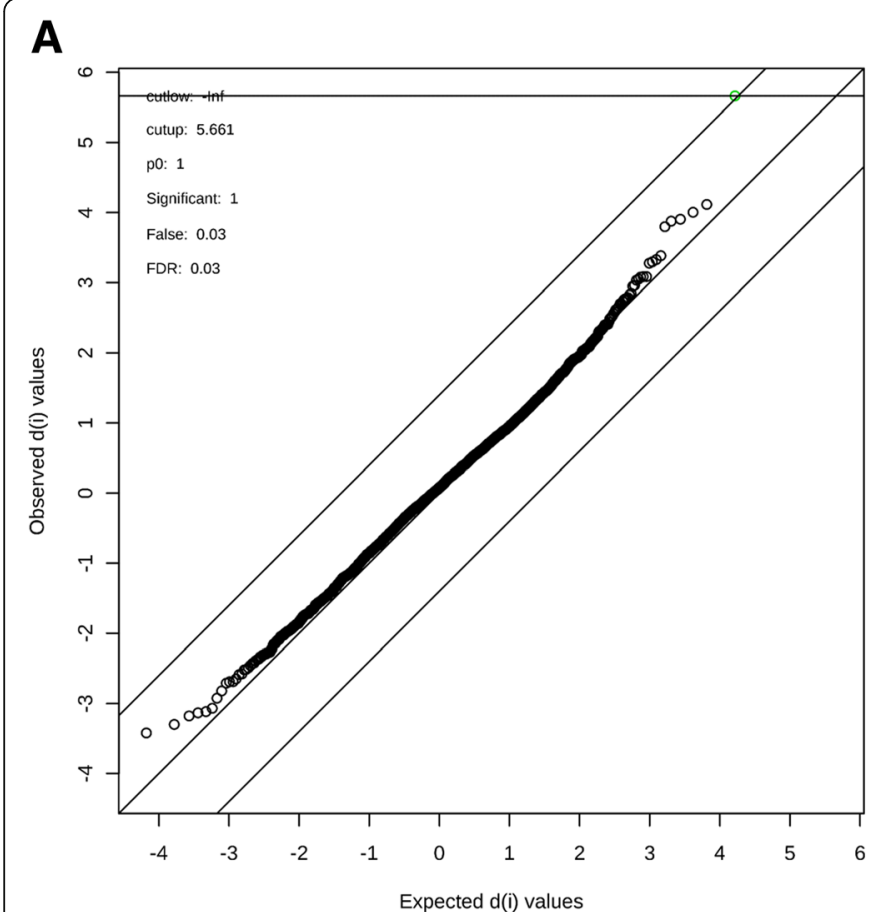

B

Fig. 2 Univariate differential analysis of features from bovine metabolome. a Univariate analysis corrected by multiple tests (SAM-FDR) results for positive mode features. $\mathbf{b}$ The difference of abundance between the HFE and LFE groups for the $\mathrm{m} / \mathrm{z} 183.1670$ peak with a retention time of $4.00 \mathrm{~min}$ (positive mode; SAM-FDR $\leq 0.05$ )

$p$-values (Fig. 3) and common features, suggesting that both networks belong to the same molecular signature.

We were able to identify 7 molecules from the co-expressed modules through Mummichog prediction: Retinal, Progesterone, Stearic acid, Vomifoliol, 2,3 Dihydroflavone, Limonoate and Phytanic acid. Interestingly, all these molecules have higher levels in LFE animals which are in accordance with the modules being positively associated with RFI. In addition, mummichog software predicted two molecules from the retinol pathway significantly associated with FE: a higher level of Retinal and lower level of Retinoate (C00777) in LFE, which implies the enzymes aldehyde oxidase and retinal dehydrogenase (that convert Retinal to Retinoate) as probably less active/expressed in LFE animals. This result is in accordance with Zhao and colleagues [37] who demonstrate vitamin A (VA) metabolism is important for feed efficiency in pigs as key genes of VA metabolism such as ALDH1A2 and CYP1A1 are upregulated in the liver of HFE animals.
Also, in two transcriptome studies, the retinol pathway was upregulated in the liver of high-RFI Jersey steers [38] and over-represented in the small intestine from high intake beef steers [39]. A GWAS-study using CNV markers evidenced the RDH5 (an important gene of the retinol metabolism pathway) as a candidate gene associated with feed conversion rate in Nellore cattle [40]. Therefore, our results agreed with the literature regarding the importance of the retinol metabolism pathway for feed efficiency in livestock animals.

Progesterone (P4) was another feature predicted in the molecular signature of FE being more present in the blood of LFE animals. Steroid hormone biosynthesis was overrepresented in the set of genes in the liver that were upregulated in the high-RFI (low FE) group of Jersey cows [38], which is in accordance with our results. Recently, P4 signaling in broiler skeletal muscle was associated with divergent feed efficiency [41]. So far, there is no consensus on the role of $\mathrm{P} 4$ on feed

Table 2 Metabolic pathways for RFI prior to the feedlot and their size on the positive mode of acquisition

\begin{tabular}{lllll}
\hline Pathway & Pathway size & Total Hits & Significant Hits & Fisher's $P$ value \\
\hline Retinol metabolism & 17 & 6 & 2 & $0.0237^{*}$ \\
Steroid hormone biosynthesis & 67 & 8 & 1 & 0.3055 \\
Arachidonic acid metabolism & 36 & 7 & 1 & 0.2725 \\
\hline
\end{tabular}


Table 3 Significant analytes predicted by mummichog

\begin{tabular}{llllll}
\hline $\mathrm{m} / \mathrm{z}$ & Compound & adduct & mass diff & $P$ value & HFE/LFE \\
\hline 267.2105 & all-trans-Retinal / 11-cis-Retinal & $\mathrm{M}-\mathrm{H} 2 \mathrm{O}+\mathrm{H}[1+]$ & 0.00017686 & $0.0075^{*}$ & Down \\
273.2233 & Retinoate & $\mathrm{M}-\mathrm{CO}+\mathrm{H}[1+]$ & 0.00213163 & $0.0019^{*}$ & Up
\end{tabular}

The mass-charge $(\mathrm{m} / \mathrm{z})$, compounds hit, mass difference, analyte $p$-value and FE group association

efficiency in livestock and further studies should be performed.

The stearic acid is a saturated acid (C18:0) and one of the end products of the fatty acid biosynthesis pathway in animals. This fatty acid was found increased in plasma of steers with least ADG in comparison with greatest ADG [42], and this result corroborates our finding of a higher level of stearic acid in the molecular signature associated with LFE animals since they had less ADG than HFE in our experiment.

From all predicted molecules, Vomifoliol, 2,3 Dihydroflavone, Limonoate and Phytanic acid are molecules produced exclusively by bacteria or plants and not mammals. The higher presence of these molecules in the blood of LFE animals could be due to higher DMI of these animals in comparison with HFE animals, allowing the higher presence of these metabolites in the blood. However, this possibility lacks further evidence since we did not evaluate the pasture DMI of these animals, i.e. feed intake before they arrive at the feedlot. From these 4 molecules, the Phytanic Acid could have a role on feed efficiency. Phytanic acid is a branched-chain fatty acid formed during the metabolism of phytol [43] by ruminal bacteria and is a known agonist for the nuclear-receptor-retinoid-X-receptor [44] and the peroxisome proliferated-activated receptor- $\alpha$ (PPAR- $\alpha$ ) [45]. These two proteins are important nuclear receptors regulating the expression of several genes in response to environmental factors (i.e. diet) and endogenous molecules. Interestingly, in rats, agonists of PPAR- $\alpha$ decreased feed

\section{A}

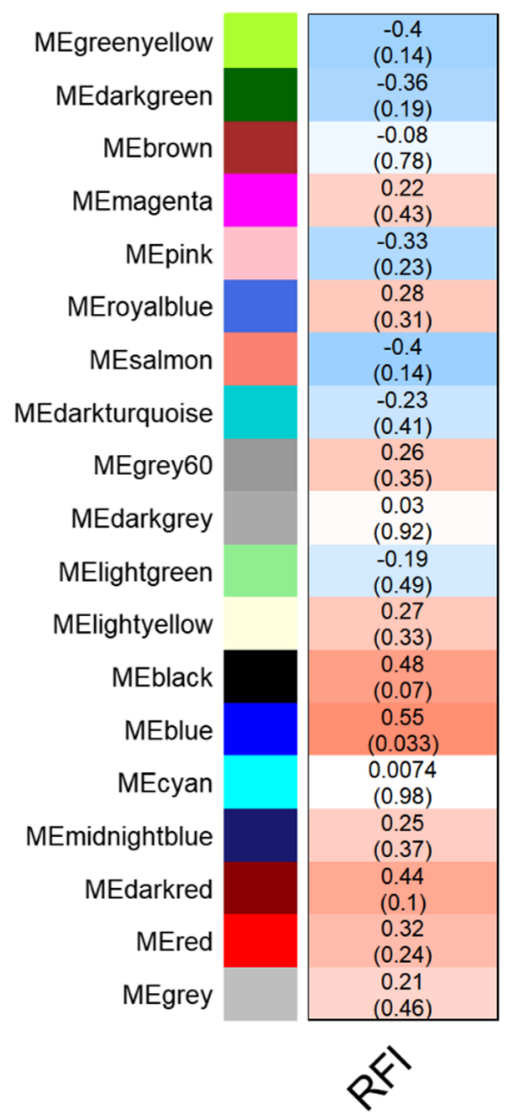

B

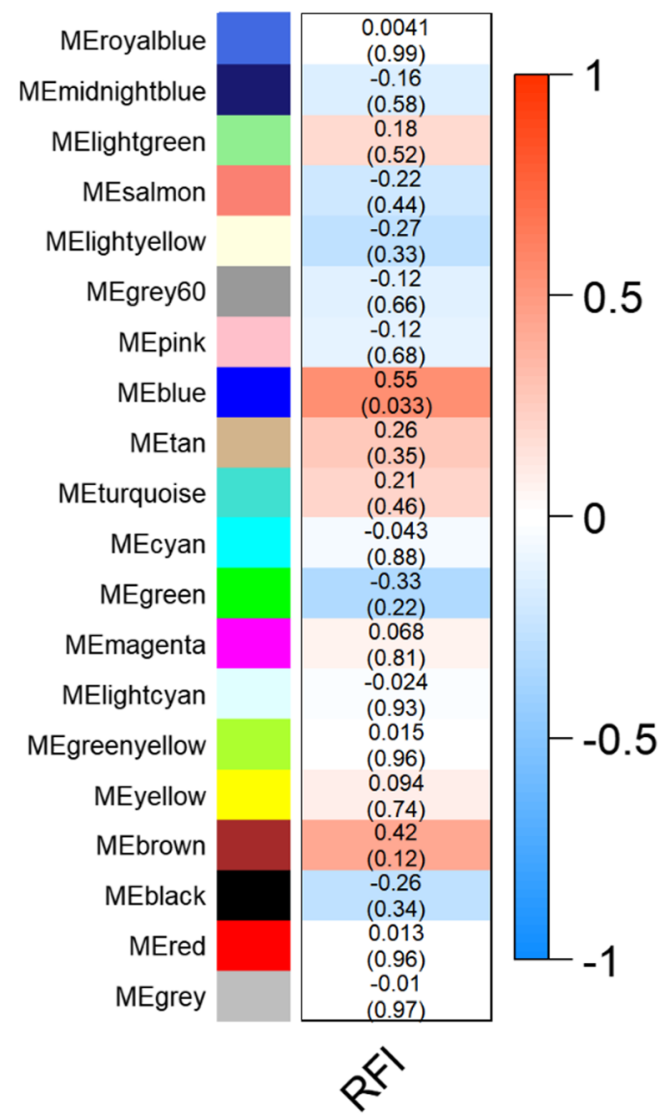

Fig. 3 Network analysis of co-expressed features in the negative and positive mode of acquisition. Pearson correlation between residual feed intake (RFI) and the module eigengenes in the negative (a) and positive (b) mode. In each line the color name of modules (ME). The number in each module is the Pearson correlation between the module and RFI; In brackets the $p$-value of the correlation 
efficiency [46], and the PPAR signalling pathway was enriched in the small intestine transcriptome analysis of high vs. low feed intake cattle [39]. Therefore, agonists of PPAR- $\alpha$ could reasonably be associated with feed efficiency in cattle, but new evidence should be provided to confirm this hypothesis.

Our integrated approach using data annotation, mummichog prediction and WGCNA co-expression analyses indicated a molecular signature enriched for biological processes previously associated with $\mathrm{FE}$. The metabolites in WGCNA modules were also predicted by mummichog, which supports the validity of the in silico network analysis since the two different analyses yielded consistent results. Therefore, we believe metabolomics based modules associated with FE possibly represent a molecular metabolic signature of FE. Although we have not yet been able to identify the majority of the features in those modules, previous studies on feed efficiency support the network analysis results. Moreover, we noted it is possible to have a molecular signature associated with a phenotype without knowing the function of the components, just by (for metabolites) tracking $\mathrm{m} / \mathrm{z}$ ratio and retention time in a standardized assay. As an example, this is the case for commercially available genomic selection in dairy cattle using DNA markers, where the majority of the markers are not functional SNPs.

In our data, we found only one feature statistically different between the FE groups: the feature with $\mathrm{m} / \mathrm{z}$ 183.1670 and RT of $4.00 \mathrm{~min}$ (positive mode) is upregulated in HFE animals. This result along with the co-expressed module provides evidence of early serum metabolome differences between high and low FE animals. Between both the positive and negative ionization modes and after quality control-based filtering, the serum metabolome of the animals in this experiment consisted of approximately 8000 features. One may expect a priori to identify more than just one different feature between high and low FE animals using such a powerful tool. Possible explanations for this result include, but are not limited to: (1) although the groups are very distinct phenotypically at the end of the experiment, their baseline metabolic profiles may have been more similar at the time when samples were collected (21 days before the beginning of the feeding trial) [9]; (2) the FE was estimated for feedlot performance and not for pasture grazing; at the time of sampling all animals were still on pasture conditions, which may yield more similar metabolic phenotypes than a high grain diet; (3) the animals were clinically healthy over the whole experiment. Thus, no major physiological disturb could lead to large metabolome difference between the FE groups; (4) the number of sampled animals (8 animals per group) could limit the statistical power [47] for these outbred, genetically different animals that may have high baseline diversity in metabolic profiles. To address this last issue, one of our ongoing projects is to validate these results in a cohort with more animals, to develop a future technology help establish a framework for future for FE prediction.

\section{Conclusion}

The conclusion from this work is the detection of a molecular signature for feed efficiency of beef cattle based on untargeted metabolomics. This molecular signature indicated the vitamin A metabolism pathway as one of the important pathways for this phenotype.

\section{Additional files}

Additional file 1: Experiment information of animals including group, birth, days of life at before feedlot ( -21 days), father, residual feed intake and residual feed intake adjusted by age as a covariate. The FE groups had different ages $(P<0.05)$. To perform the Network analysis, the phenotype was adjusted by age, fitted as a covariate. (CSV 905 bytes)

Additional file 2: Important features in blue module in negative acquisition mode. Mass/charge ratio (m/z); Gene significance (GS); Module Membership (MM); Feature connectivity within the module (Kwithin); Adducts; Highest abundance group; Adducts; Matched Compound (KEGG by mummichog); Mass difference between $\mathrm{m} / \mathrm{z}$ and matched compound. (CSV 6 kb)

Additional file 3: Important features in blue module in positive acquisition mode. Mass/charge ratio (m/z); Gene significance (GS); Module Membership (MM); Feature connectivity within the module (Kwithin); Adducts; Highest abundance group; Adducts; Matched Compound (KEGG by mummichog); Mass difference between $\mathrm{m} / \mathrm{z}$ and matched compound. (CSV $3 \mathrm{~kb}$ )

\section{Abbreviations}

ADG: Average daily gain; ALDH1A2: Aldehyde Dehydrogenase 1 Family Member A2 gene; CNV: Copy-number variation; CYP1A1: Cytochrome P450 Family 1 Subfamily A Member 1 gene; DMI: Dry matter intake; FE: Feed efficiency; HFE: High feed efficiency group; LFE: Low feed efficiency group; NEG: Negative mode acquisition; P4: Progesterone; POS: Positive mode acquisition; PPARa: Peroxisome proliferated-activated receptor-alpha; RDH5: 11-cis retinol dehydrogenase 5 gene; RFI: Residual feed intake; VA: Vitamin A

\section{Acknowledgments}

The authors are thankful to Professors Paulo Roberto Leme and Saulo da Luz e Silva for their kind help on the feedlot experiment.

\section{Funding}

All the experiments were financially supported by "Fundação de Amparo à Pesquisa do Estado de São Paulo", Brazil (FAPESP grants: 2014/02493-7 and 2014/07566-2). FAPESP have no role in the design of the study and collection, analysis, and interpretation of data and in writing the manuscript. FAPESP also contributed with a scholarship to FJN (grant 2015/01059-4).

\section{Availability of data and materials}

The datasets used and/or analyzed during the current study are available from the corresponding author on reasonable request.

\section{Authors' contributions}

FJN performed the sample preparation, bioinformatics, data analysis and wrote the manuscript; PRLP contributed to the metabolomics protocols and data analysis; PAA conducted the in vivo experiment, sample collection and WGCNA initial analysis; RD contributed with the mummichog initial analysis. $\mathrm{AHI}$ generates the mass-spectrometry raw data and assisted with data quality assurance; JBSF contributed with the experimental design; MPS contributed with quality control procedure and Metaboanalyst analysis. HF was the 
overall project leader who conceived and conducted this study and were the advisor of FJN. All authors have read, made suggestions and approved the final manuscript.

\section{Ethics approval}

All animal protocols were approved by the Institutional Animal Care and Use Committee of Faculdade de Zootecnia e Engenharia de Alimentos, Universidade de São Paulo (FZEA-USP - protocol number 14.1.636.74.1). The animals belonged to FZEA-USP.

\section{Consent for publication}

Not applicable.

\section{Competing interests}

The authors declare they have no competing interests.

\section{Publisher's Note}

Springer Nature remains neutral with regard to jurisdictional claims in published maps and institutional affiliations.

\section{Author details}

'Department of Veterinary Medicine, School of Animal Science and Food Engineering, University of Sao Paulo, Av. Duque de Caxias Norte n`225, Pirassununga 13635-900, Sao Paulo, Brazil. ${ }^{2}$ School of Chemical \& Biomolecular Engineering, Georgia Institute of Technology, Atlanta, Georgia USA. ${ }^{3}$ Apex Science, Campinas, Sao Paulo, Brazil.

\section{Received: 20 August 2018 Accepted: 21 December 2018} Published online: 07 January 2019

\section{References}

1. Alexandratos N, Bruinsma J. World agriculture towards 2030/2050: the 2012 revision. ESA Working paper No. 12-03 FAO. 2012.

2. Eisler MC, Lee MRF, Tarlton JF, Martin GB, Beddington J, Dungait JAJ, et al. Agriculture: steps to sustainable livestock. Nature. 2014;507:32-4. https://doi. org/10.1038/507032a.

3. Godfray HCJ, Beddington JR, Crute IR, Haddad L, Lawrence D, Muir JF, et al. Food security: the challenge of feeding 9 billion people. Science. 2010;327:812-8

4. Herrero M, Havlik P, Valin H, Notenbaert A, Rufino MC, Thornton PK, et al. Biomass use, production, feed efficiencies, and greenhouse gas emissions from global livestock systems. Proc Natl Acad Sci. 2013;110:20888-93.

5. Arthur JPF, Herd RM. Residual feed intake in beef cattle. Rev Bras Zootec 2008;37:269-79.

6. Koch RM, Swiger LA, Chambers D, Gregory KE. Efficiency of feed use in beef cattle. J Anim Sci. 1963;22:486-94.

7. Arthur PF, Archer JA, Richardson EC, Herd RM. Potential for selection to improve efficiency of feed use in beef cattle: a review. Aust J Agric Res. 1999:50:147.

8. Herd RM, Arthur PF. Physiological basis for residual feed intake. J Anim Sci. 2009:87:14. https://doi.org/10.2527/jas.2008-1345.

9. Alexandre PA, Kogelman LJA, Santana MHA, Passarelli D, Pulz LH, FantinatoNeto $P$, et al. Liver transcriptomic networks reveal main biological processes associated with feed efficiency in beef cattle. BMC Genomics. 2015;16:1073.

10. Dunn WB, Broadhurst D, Begley P, Zelena E, Francis-Mclntyre S, Anderson N, et al. Procedures for large-scale metabolic profiling of serum and plasma using gas chromatography and liquid chromatography coupled to mass spectrometry. Nat Protoc. 2011:6:1060-83.

11. Becker S, Kortz L, Helmschrodt C, Thiery J, Ceglarek U. LC-MS-based metabolomics in the clinical laboratory. J Chromatogr B. 2012:883884:68-75

12. Rohart F, Paris A, Laurent B, Canlet C, Molina J, Mercat MJ, et al. Phenotypic prediction based on metabolomic data for growing pigs from three main European breeds. J Anim Sci. 2012;90:4729-40.

13. Lu J, Boeren S, van Hooijdonk T, Vervoort J, Hettinga K. Effect of the DGAT1 K232A genotype of dairy cows on the milk metabolome and proteome. J Dairy Sci. 2015;98:3460-9.

14. Melzer N, Wittenburg D, Repsilber D. Integrating Milk metabolite profile information for the prediction of traditional Milk traits based on SNP information for Holstein cows. PLoS One. 2013;8:e70256.
15. Klein MS, Buttchereit N, Miemczyk SP, Immervoll A-K, Louis C, Wiedemann S, et al. NMR Metabolomic analysis of dairy cows reveals Milk Glycerophosphocholine to Phosphocholine ratio as prognostic biomarker for risk of ketosis. J Proteome Res. 2012:11:1373-81.

16. Widmann P, Reverter A, Weikard R, Suhre K, Hammon HM, Albrecht E, et al. Systems biology analysis merging phenotype, Metabolomic and genomic data identifies non-SMC Condensin I complex, subunit G (NCAPG) and cellular maintenance processes as major contributors to genetic variability in bovine feed efficiency. PLoS One. 2015;10:e0124574.

17. Karisa BKK, Thomson J, Wang Z, Li C, Montanholi YRR, Miller SPP, et al. Plasma metabolites associated with residual feed intake and other productivity performance traits in beef cattle. Livest Sci. 2014;165:200-11. https://doi.org/10.1016/j.livsci.2014.03.002.

18. Clemmons BA, Mihelic RI, Beckford RC, Powers JB, Melchior EA, McFarlane $\mathrm{ZD}$, et al. Serum metabolites associated with feed efficiency in black angus steers. Metabolomics. 2017;13:147.

19. Lu D, Miller S, Sargolzaei M, Kelly M, Vander Voort G, Caldwell T, et al. Genome-wide association analyses for growth and feed efficiency traits in beef cattle. J Anim Sci. 2013:91:3612-33.

20. Crowley JJ, McGee M, Kenny DA, Crews DH, Evans RD, Berry DP. Phenotypic and genetic parameters for different measures of feed efficiency in different breeds of Irish performance-tested beef bulls. J Anim Sci. 2010;88:885-94.

21. Arthur PF, Archer JA, Johnston DJ, Herd RM, Richardson EC, Parnell PF. Genetic and phenotypic variance and covariance components for feed intake, feed efficiency, and other postweaning traits in Angus cattle. J Anim Sci. 2001;79:2805-11.

22. Liu MF, Goonewardene LA, Bailey DRC, Basarab JA, Kemp RA, Arthur PF, et al. A study on the variation of feed efficiency in station tested beef bulls. Can J Anim Sci. 2000;80:435-41.

23. Mota LFM, Bonafé CM, Alexandre PA, Santana MH, Novais FJ, Toriyama E, et al. Circulating leptin and its muscle gene expression in Nellore cattle with divergent feed efficiency. J Anim Sci Biotechnol. 2017;8:71.

24. Tuck MK, Chan DW, Chia D, Godwin AK, Grizzle WE, Krueger KE, et al. Standard operating procedures for serum and plasma collection: early detection research network consensus statement standard operating procedure integration working group. J Proteome Res. 2009:8:113-7.

25. Cleveland WS. Robust locally weighted regression and smoothing scatterplots. J Am Stat Assoc. 1979;74:829-36.

26. Sumner LW, Amberg A, Barrett D, Beale MH, Beger R, Daykin CA, et al. Proposed minimum reporting standards for chemical analysis. Metabolomics. 2007:3:211-21.

27. Xia J, Wishart DS. Using MetaboAnalyst 3.0 for Comprehensive Metabolomics Data Analysis. In: Current Protocols in Bioinformatics. Hoboken, NJ: John Wiley \& Sons, Inc.; 2016. 14.10.1-14.10.91.

28. Durbin BP, Hardin JS, Hawkins DM, Rocke DM. A variance-stabilizing transformation for gene-expression microarray data. Bioinformatics. 2002; 18(Suppl 1):S105-10.

29. van den Berg RA, Hoefsloot HC, Westerhuis JA, Smilde AK, van der Werf MJ. Centering, scaling, and transformations: improving the biological information content of metabolomics data. BMC Genomics. 2006;7:142.

30. Tusher VG, Tibshirani R, Chu G. Significance analysis of microarrays applied to the ionizing radiation response. Proc Natl Acad Sci. 2001;98:5116-21.

31. Langfelder $P$, Horvath SWGCNA. An R package for weighted correlation network analysis. BMC Bioinformatics. 2008;9:559.

32. Zhang B, Horvath S. A General framework for weighted gene co-expression network analysis. Stat Appl Genet Mol Biol. 2005;4:Article17.

33. Fukushima A, Kusano M, Redestig H, Arita M, Saito K. Metabolomic correlation-network modules in Arabidopsis based on a graph-clustering approach. BMC Syst Biol. 2011;5:1.

34. Chen Y-C, Guo Y-F, He H, Lin X, Wang X-F, Zhou R, et al. Integrative analysis of genomics and transcriptome data to identify potential functional genes of BMDs in females. J Bone Miner Res. 2016:31:1041-9.

35. Li S, Park Y, Duraisingham S, Strobel FH, Khan N, Soltow QA, et al. Predicting network activity from high throughput metabolomics. PLoS Comput Biol. 2013;9:e1003123.

36. Hudson NJ, Dalrymple BP, Reverter A. Beyond differential expression: the quest for causal mutations and effector molecules. BMC Genomics. 2012;13: 356. https://doi.org/10.1186/1471-2164-13-356.

37. Zhao $Y$, Hou Y, Liu F, Liu A, Jing L, Zhao C, et al. Transcriptome analysis reveals that vitamin a metabolism in the liver affects feed efficiency in pigs. G3 (Bethesda). 2016;6(11):3615-24. 
38. Salleh MS, Mazzoni G, Höglund JK, Olijhoek DW, Lund P, Løvendahl P, et al. RNA-Seq transcriptomics and pathway analyses reveal potential regulatory genes and molecular mechanisms in high- and low-residual feed intake in Nordic dairy cattle. BMC Genomics. 2017;18:258.

39. Lindholm-Perry AK, Butler AR, Kern RJ, Hill R, Kuehn LA, Wells JE, et al. Differential gene expression in the duodenum, jejunum and ileum among crossbred beef steers with divergent gain and feed intake phenotypes. Anim Genet. 2016;47:408-27.

40. de Almeida Santana MH, Junior GAO, Cesar ASM, Freua MC, da Costa Gomes R, da Luz e Silva S, et al. Copy number variations and genome-wide associations reveal putative genes and metabolic pathways involved with the feed conversion ratio in beef cattle. J Appl Genet. 2016;57:495-504.

41. Bottje W, Kong B-W, Reverter A, Waardenberg AJ, Lassiter K, Hudson NJ. Progesterone signalling in broiler skeletal muscle is associated with divergent feed efficiency. BMC Syst Biol. 2017:11:29.

42. Artegoitia VM, Foote AP, Lewis RM, Freetly HC. Rumen fluid metabolomics analysis associated with feed efficiency on crossbred steers. Sci Rep. 2017;7:2864

43. Hellgren LI. Phytanic acid-an overlooked bioactive fatty acid in dairy fat? Ann N Y Acad Sci. 2010;1190:42-9.

44. Kitareewan S, Burka LT, Tomer KB, Parker CE, Deterding L, Stevens RD, et al. Phytol metabolites are circulating dietary factors that activate the nuclear receptor RXR. Mol Biol Cell. 1996;7:1153-66.

45. Ellinghaus P, Wolfrum C, Assmann G, Spener F, Seedorf U. Phytanic acid activates the peroxisome proliferator-activated receptor a (PPARa) in sterol carrier protein 2-/ sterol carrier protein $x$-deficient mice. J Biol Chem. 1999; 274:2766-72.

46. Larsen PJ, Jensen PB, Sorensen RV, Larsen LK, Vrang N, Wulff EM, et al. Differential influences of peroxisome proliferator-activated receptors and on food intake and energy homeostasis. Diabetes. 2003;52:2249-59.

47. Brereton RG, Lloyd GR. Partial least squares discriminant analysis: taking the magic away. J Chemom. 2014;28:213-25.

Ready to submit your research? Choose BMC and benefit from:

- fast, convenient online submission

- thorough peer review by experienced researchers in your field

- rapid publication on acceptance

- support for research data, including large and complex data types

- gold Open Access which fosters wider collaboration and increased citations

- maximum visibility for your research: over $100 \mathrm{M}$ website views per year

At $\mathrm{BMC}$, research is always in progress.

Learn more biomedcentral.com/submissions 